\title{
Design of Cross-culture Business Graphic Symbol Communication Application
}

\author{
Wei Wang \\ International Business School \\ Yunnan University of Finance and Economic \\ Kunming, China \\ 1305367332@qq.com
}

\author{
Yuhui Feng* \\ International Business School \\ Yunnan University of Finance and Economic \\ Kunming, China \\ feng@ynufe.edu.cn
}

\begin{abstract}
With the development of The Belt and Road construction, the business communication between those Countries which located in GMS has been more frequent and deeper. Thus, intercultural network communication is urgent demanded indeed. However, the so-called intelligent translation software does not fully cover many minority languages in GMS, and due to the limitation of education, English is difficult to be used by businessman as a general language in this area. So using the more intuitive graphic symbols to communicate can be a method worth exploring. Therefore, it is necessary to develop an instant messaging(IM) App based on commercial graphic symbols, and use the results of graphic symbol communication, or carry out experiment in it. This paper is aimed to elaborate the function, framework design and practical effect of the APP.
\end{abstract}

Keywords-graphic symbol communication; IM APP framework design; intercultural business exchange

\section{INTRODUCTION}

According to the method of multimodal discourse analysis, the text can be constructed by sound, graphics and all kinds of normative languages, and express an accurate meaning to a certain extent. According to Hongxiu Fu's viewpoint, it can be indicate by the intersymbol complementarity of the corpus that the intersymbol complementation of the corpus shows that the emoticons belong to the interpersonal function oriented image, and the relatively high frequency of the two inter symbolic meanings of repetition and synonym indicates that the emoticons and languages complement each other to maintain and support the same topic [1].

The so-called intersymbol complementarity, that is, two or more different symbol resources can be used together effectively [2]. It is a great concern for GMS, a region with many minority languages and frequent business activities. Language barriers are one of the great obstacles to information sharing and e-commerce development in sub regional countries and regions [3]. This obstacle is particularly prominent in the GMS. The relevant data show that the official and quasi official languages used only in the GMS are 9 languages, such as Chinese, Vietnamese, French, Khmer, Laotian, Burmese and Thai. Besides, there are more ethnic languages in the areas bordering China and in ethnic areas such as Yunnan and Guangxi. Therefore, the language barrier existing in the cross border e-commerce websites of the GMS is the most fundamental and urgent problem to solve in its development process. And there are many ethnic groups in every country, all ethnic groups have their own languages, and there are 27 kinds of ethnic languages in Yunnan province [4]. Obviously, there will be no specialized software to develop translation functions for these ethnic languages because of the limited usage and limited scope. With regard to educational resources in the region, it is also impractical for small and medium enterprises and their business people to communicate in English. Multimodal discourse can complement the linguistic deficiencies, especially those which are difficult to express.

In the category of cognitive psychology, experts believe that the process of processing the input information by a computer is very similar to the process of cognition when human beings accept external information [5]. The whole process is as follows:

- Input external information;

- Encoding external information;

- To form a mapping in a computer (or brain);

- Decoding the map by a certain algorithm (or experience and knowledge);

- Explain the specific meaning of external information.

Let's use a common scenario to analyze the process in detail. Suppose we are watching a pantomime on the stage, the whole process is not supported by any language, it is entirely dependent on the face expression of the actors, the movements of the body and the picture of the stage environment. Applying the above cognitive process paradigm, the specific process is as follows:

- The entire stage is completely input to the viewer's brain with visual signals;

- The audience's brain packs the entire stage picture at this time point;

- The audience's brain abstracts every part of the stage (actors' movements, props and backgrounds);

*Corresponding author 
- Audiences' brains understand and visualize each part through their past life experience accumulation and knowledge;

- The viewer's brain connects and integrates all parts, and then understands the meaning of the whole stage.

We find that audiences can fully understand the plot of a pantomime based solely on simple visual signals. This not only contains objective and factual understanding (such as the action of the actors, the events in the story, etc.), and even the inner feelings of the characters in the deeper levels of the play. Accordingly, the expression of graphic symbols in the Internet is the reversion of the "dumb" expression process, but its dialogue platform is converted from the stage to the Internet, and each graphic symbol is an abstraction of the face expression and body movements of the actors and background images. It can be seen that the communication based on graphic symbols is entirely logical.

Menglan Qiu has recorded an experiment in her paper "Cross-culture Business Communication by Emoji in GMS". The researchers divided the volunteers from Laos, Burma, Thailand and Vietnam into four groups as the experimental subjects. The four groups of emoticons expressing different emotions were presented randomly to the subjects. The subjects responded the meaning expressed by the group of emoticons to researchers. The experimental results show that the correct recognition rate of emoticons expressed by each group is more than $80 \%$ and the correct recognition rate of angry and sad is up to $95.83 \%$ and $92.26 \%$ respectively. This is enough to illustrate the feasibility of using graphic symbols to intercultural communication on the Internet in reality [6].

At present, the mainstream IM APPs, such as QQ, WeChat, MSN and so on, has used emoticons as an auxiliary expression tool. But as long as we go deep into the analysis, we can see that the design and understanding process of these emoticons all follow the framework shown in Fig. 1.

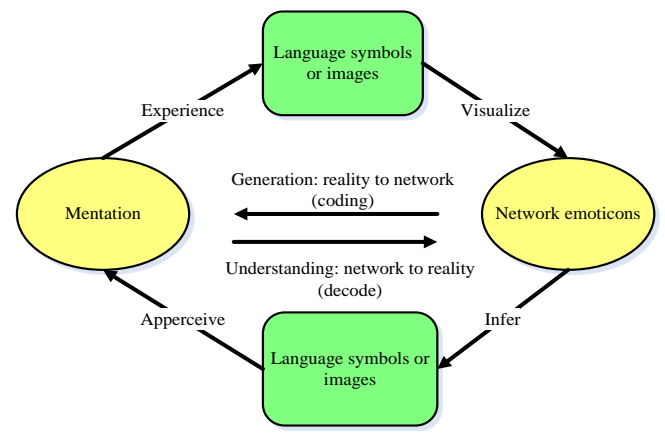

Fig. 1. The process of design and understanding of network emoticons.

That is to say, the contemporary emoticons that are popular on the Internet can only be used to express subjective emotions, but do not add more graphic symbols of objective facts. This is the reason why people call it "emoticons" and rarely call it "graphic symbols". As a result, the situation that these emoticons can be applied to is very limited, almost expressing only the emotional content in daily social situations. In fact, this is because the IM APPs that use these emoticons, its original location is daily social contact and entertainment, and its goal is only convenient and entertaining. Even though the APPs such as WeChat, MSN and QQ classified emoticons, but it is only a classification of all kinds of cartoon images or emotional tendencies, only a metaphysical partition that essentially fails to get out of the monotonous category of "emotion" [7]. In South and Southeast Asia, businesses are frequent, culturally diverse and difficult to communicate, so an instant messaging APP which can express more objective content, classifications and more use scenes(business scenes in particular) with graphic symbols will be very in line with demand.

\section{APPLICATION ARCHITECTURE AND EXTENSION OF INSTANT MESSAGING}

\section{A. The Architecture of the Current Major Instant Messaging} Applications

\begin{tabular}{|c|c|c|c|c|}
\hline Clients & Mobile terminal & App & \multicolumn{2}{|c|}{ Customer service system } \\
\hline Clients' API & Android SDK & IOS SDK & \multicolumn{2}{|c|}{ WebSocket } \\
\hline Access layer & TCP access & \multicolumn{2}{|c|}{ WebSocket access } & HTTP access \\
\hline \multirow{2}{*}{ Logic layer } & $\begin{array}{c}\text { Offline } \\
\text { message }\end{array}$ & $\begin{array}{l}\text { Message } \\
\text { push }\end{array}$ & $\begin{array}{c}\text { Login } \\
\text { authorization }\end{array}$ & \\
\hline & Group chat & News report & Organization & \\
\hline Persistence layer & Redis caching & & & File server \\
\hline
\end{tabular}

Fig. 2. WeChat APP design framework.

Take WeChat as an example, the design framework of popular IM software at present is shown in Fig. 2. All levels are described as follows:

- Clients: the focus of the clients is mobile terminals, supporting IOS/Android system, including IM App, embedded melon App with message function, and may also access customer service system in the future.

- Clients' API: for the TCP protocol, SDK is provided to IOS/Android development. For the HTML5 page, the WebSocket interface is provided.

- Access layer: the main task of access layer is to maintain massive user connection (access), attack protection, convert mass connections into a small number of TCP connections and communicate with logical layer.

- Logical level: the logic layer is responsible for the core logic realization of all functions of IM system. It includes single chat $(\mathrm{C} 2 \mathrm{C})$, sending $(\mathrm{C} 2 \mathrm{~S})$, push(S2C), group chat $(\mathrm{C} 2 \mathrm{G})$, offline message, login authorization, organization tree and so on.

- Storage layer: the storage layer is responsible for caching or storing IM system related data, mainly including user status and routing (caching), message data (MySQL, also NoSql, or MangoDB), file data (file server).

The specific data transfer aspect is as shown in the following fig. 3. : 


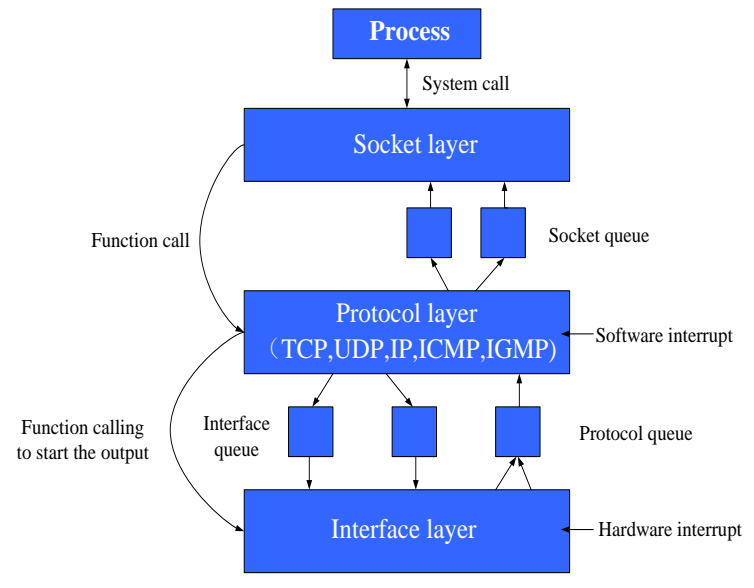

Fig. 3. Data transfer schematic.

- Each socket in the socket layer has an input queue and an output queue;

- Each protocol in the protocol layer has one input queue and output queue;

- Each interface in the interface layer has an input queue and output queue.

Among them, the specific socket communication process is as follows:

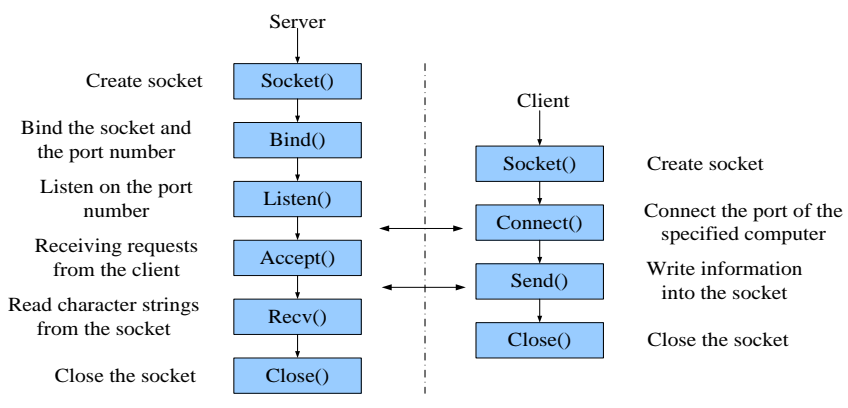

Fig. 4. .Socket communication process.

Several key functions in the socket process are explained:

- Socket_create(\$net parameter1, \$stream parameter2, \$protocol parameter3)

Function: create a socket, that is a network data stream.

Return: a socket or false, the parameter error sends a E_WARNING.

- Socket_connect(\$socket parameter1, \$ip parameter2, \$port parameter3)

Function: connect a socket.

Return: true or false.

Parameter1: the return value of socket_create.

Parameter2: the IP address.

Parameter3: the port number.
- Socket_bind(\$socket parameter1, \$ip parameter2, \$port parameter3)

Function: bind a socket.

Return: true or false.

Parameter1: the return value of socket_create.

Parameter2: the IP address.

Parameter3: the port number.

- Socket-listen(\$socket parameter1, \$backlog parameter2)

Function: listen to a socket.

Return: true or false.

Parameter1: the return value of socket_create.

Parameter2: maximum monitor number of sockets.

- Socket_accept(\$socket parameter)

Function: receive the resource information of the socket.

Return: the information resource of the socket or false.

Parameter: the return value of socket_create.

- Socket_read(\$socket parameter1, \$length parameter2)

Function: read the resource information of the socket. false.

Return: convert socket resources into string information or

Parameter1: the return value of socket-create or socket_accept.

Parameter2: the length of the string.

- Socket_write(\$socket parameter1, \$msg parameter2, \$strlen parameter3)

Function: write the data into the socket.

Return: the byte length of the string or false.

Parameter1: the return value of socket_create or socket_accept.

Parameter2: a string.

Parameter3: the length of the string.

- Socket_close(\$socket parameter)

Function: close the socket.

Return: true or false.

Parameter: the return value of socket_create or socket_accept.

The function, communication and logic framework of WeChat based IM APP is described above. However, such IM APP does not make the classification and calling module of graphic symbols more scientific, detailed and more suitable for use. This is far from meeting the needs of GMS area for cross-cultural communication with graphical symbols. Therefore, researchers have expanded the modules designed 
for graphical symbols exchange on the basis of these IM APPs architectures.

\section{B. An Extended Architecture for Communication of Symbols}

The system of the platform is built on the WAMP, using a server in the laboratory, developing with PHP+MySQL, using Ajax technology to interact on the web, and using AI and other design software to make a set of graphic symbols in accordance with the context, that is, the nonliteral symbols used to Express problems in communication and transactions.

As mentioned in the previous article, the APP is designed to apply more scenarios mainly in the context of business context, so in the logical architecture, a business intelligence module that specializes in scientific classification of graphic symbols is added.

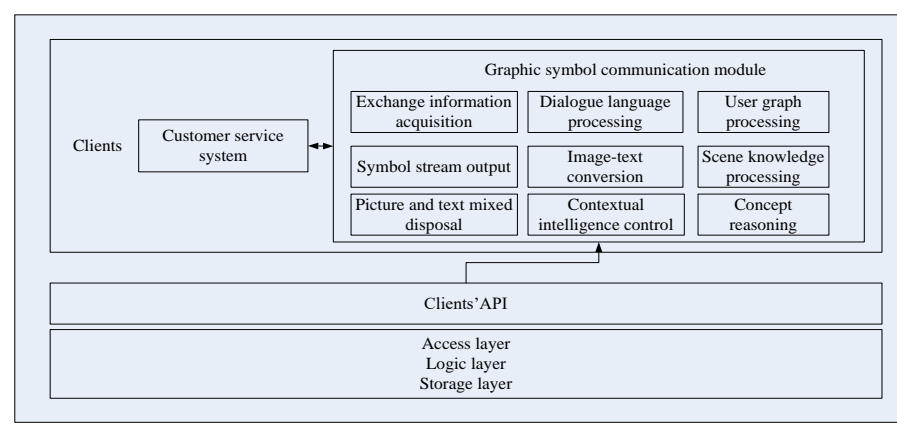

Fig. 5. An extended architecture for communication of symbols.

The main modules are as follows:

- Conversational language processing: taking out the graphical symbols which are not in the ASKII code table and processing separately;

- Image-text conversion: conversion of graphic symbols and semantic text;

- Contextual intelligence control: Based on the context constraints of a certain context;

- Scene knowledge processing: judge which business process node is, give the vocabulary of the scene, and obtain the knowledge of the current communication needs from the network, such as the currency exchange rate, weather forecast information and so on;

- User symbols processing: the size and archiving processing of user-self built graphic symbols;

- Conceptual reasoning: knowledge reasoning based on domain ontology.

The main interfaces' functions are as follows:

- Get the conversation content of the customer;

- The graphical symbols are classified and arranged in the customer service system;

- Displaying the image and text information in the dialog box of the customer service system;
- Set the format of the text and image typesetting of the customer service system.

\section{DESIGN OF GRAPHIC COMMUNICATION MODULE FOR BUSINESS INTELLIGENCE}

\section{A. Design Idea}

The design of the APP is designed to try to achieve cross cultural communication through graphic symbols in more scenes through the design and classification of more language scenes, especially in business scenarios, in order to solve the communication barriers between different countries and nations in the GMS business activities. Therefore, the main business intelligence module that distinguishes the APP from others is the "graphical symbol classification module + graphic symbol communication module". The main design ideas are as follows :

- The session contents are parsed by the session module or the graphic symbol exchange module (the APP preserves the traditional text session module, which is used to supplement the immature graphical symbol communication at the initial stage of the trial).

- Business monitoring and statistics module determines which stage the business is in the process.

- In the graphic symbol classification module, various graphic symbols are classified according to the knowledge of the scene (TABLE 1. is part of the graphical symbols of the agricultural product subcategory in the transaction category), and the users can choose according to their own usage scenes or expressions.

TABLE I. GRAPHICAL SYMBOLS OF SOME AGRICULTURAL PRODUCTS.

\begin{tabular}{|c|c|c|}
\hline Word & $\begin{array}{c}\text { Graphic s } \\
\text { ymbol }\end{array}$ & Remark \\
\hline Eggs & $\begin{array}{l}\text { GBT 10001.5-2006 Use graphical symbo } \\
\text { 1s for public information Part5 Shopping } \\
\text { symbols }\end{array}$ \\
\hline $\begin{array}{c}\text { Frain } \\
\text { Fruit }\end{array}$ & $\begin{array}{l}\text { GBT 10001.5-2006 Use graphical symbo } \\
\text { 1s for public information Part5 Shopping } \\
\text { symbols }\end{array}$ \\
$\begin{array}{c}\text { Vegeta } \\
\text { ble }\end{array}$ & $\begin{array}{l}\text { GBT 10001.5-2006 Use graphical symbo } \\
\text { 1s for public information Part5 Shopping } \\
\text { symbols }\end{array}$ \\
\hline $\begin{array}{c}\text { Dairy } \\
\text { product } \\
\text { s }\end{array}$ & $\begin{array}{l}\text { GBT 10001.5-2006 Use graphical symbo } \\
\text { 1s for public information Part5 Shopping } \\
\text { symbols }\end{array}$ \\
\hline
\end{tabular}

- Conversational module and graphic symbol exchange module will make a text-image conversion of user's conversation content.

- Combined with the characteristics of application scenarios, the constraints of language scene are 
introduced into the interface design to enable users to feel the context in conversation.

\section{B. Function and Use Scene}

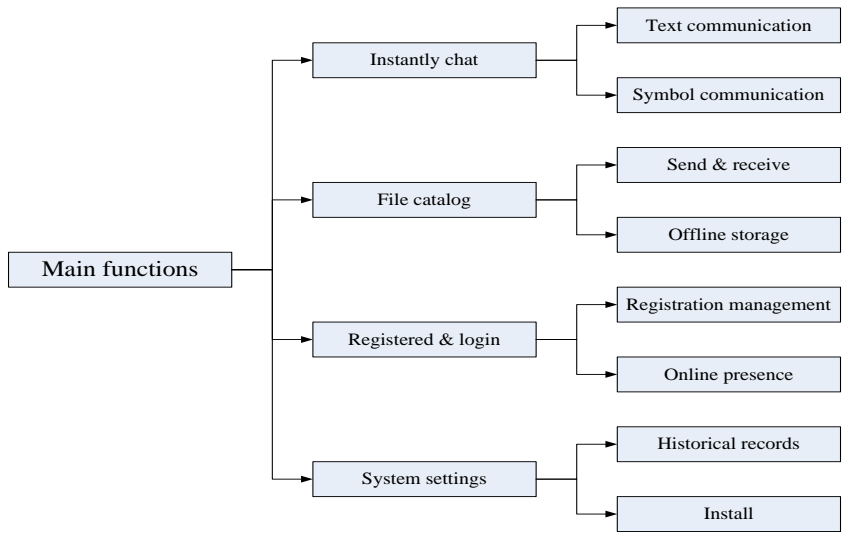

Fig. 6. Software functional framework diagram

The general functional framework of the APP is shown in Figure 6. Limited to the length of the article, here only describes the part of the exchange of symbols in the real-time conversation function.

As a social phenomenon, language is unquestionable. People acquired it in the learning and communication process. As a common view product of a particular group of people, it is actually a symbol system, a tool commonly used by people to communicate with one another. However, because of the complexity and permanence of the evolution and development of the human population, the variety of language types has become an obstacle to the communication between people and people, regions and regions in the process of global integration today. The rise of graphic symbols provides a good choice to dissolve the differences.

Firstly, the symbolization of the network uses a common consensus symbol to convey the original language cognition of the children, because the emotional expression of childhood has reached a high degree of consistency throughout the whole nature, and even the two groups with great cultural differences are bound to achieve good emotional expression. Communication, and pictures, the first human understanding of language symbols, can convey this commonality in the best way [9].

Secondly, with the growth of age and the influence of the surrounding environment, the specific language regional characteristics become the main body in the process of individual language communication. However, this has almost become the biggest obstacle of language communication in the process of global integration. Therefore, it is the direct task of network language symbols to sum up the common points in culture and avoid language culture conflicts and obstacles.

In the process of network language communication, except for the specific directivity of the characters, such as video and audio, and the way of direct communication in face to face, most of the cases belong to written language. Therefore, in addition to the retention of text conversation modules, the APP's graphic symbols are more scientifically divided according to ontology, level category hierarchy theory and relevance theory. They can be divided into five main classes: grammar, emotion, scene, trade and space-time.

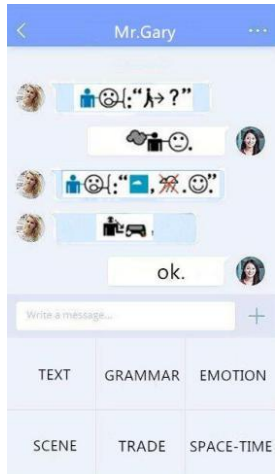

Fig. 7. Using interface diagram

\section{1) Grammar class}

This class is a category closest to the use of a word language. When a user sends a graphic symbol, it is sent in the order of "subject + predicate + object (optional)".For example, the conversation in the first dialog box in Figure 6 is the grammatical emoticons:

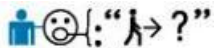

The subject (the humanoid symbol) + predicate (ask where to go): we can see that every graphic symbol of the whole message has a text prototype corresponding to it, and fully conforms to the most basic subject-predicate grammar. As a matter of fact, this is also a symbol breaking the single emotional expression and adding more objective facts, for example, the action expressed in the predicate. When we need to ask some daily actions, emoticons in grammar categories can basically meet most of the conversation requirements.

\section{2) Emotion class}

The emoticons itself are an image symbol that appears in order to adapt to the mode of online communication. Researchers believe that online chatting is between telephone conversation (or voice chat) and text correspondence, it combines two characteristics of face-to-face conversation and telephone calling. Although the text breaks through the spatial and temporal constraints of interpersonal communication, it also filters out "field information" in face-to-face communication, which refers to a large number of nonverbal information, such as emotions. Non verbal information occupies a large proportion in successful interpersonal communication. It can provide important supplementary information such as role, emotion, voice and so on. The information of this kind of inner activity is very important. Therefore, this APP preserves the emotional graphic symbols commonly found in the social software to express a lot of subtext which is difficult to express.

\section{3) Scene class}

As we often do in oral language, we often do not follow the established grammar. The setting of the scene class is mainly to cater for the nonstandard expression of daily life and social interaction. According to the different scenes, users can freely 
organize the graphic symbols that conform to the situation to achieve the purpose of more flexible expression.

\section{4) Trade class}

Because the design of this APP is mainly to solve the cross cultural communication barriers in the business activities of the GMS, a special category of graphic symbols is set up to express the content of the transaction more specifically.

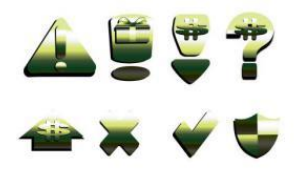

Fig. 8. Example of the symbols of the trade class

In this class, symbols such as money, agricultural products, electronic products and daily necessities are now dominated. Later, symbols such as the expression of the details of the logistics and more trade areas will be expanded.

5) Space-time class

This class mainly expresses symbols in space and time, which is designed to make it more convenient for users to express their place and time, and to help express the content of other classes more accurately.

\section{TRIAL RECORD}

In this trial, 20 students from South and Southeast Asia were invited, divided into 5 groups, each group of 4 people, using various types of emoticons on the app to communicate in real time. The users of each group were a foreign student and a researcher respectively. The exchange content was a dialogue text prepared by the researchers. The researchers recorded the completion of the text in the process of communication and calculated the average value.

TABLE II. TRIAL RECORD

\begin{tabular}{cccccc}
\hline Record & grammar & emotion & scene & business & space-time \\
\hline group1 & 90.46 & 88.60 & 87.35 & 88.32 & 82.37 \\
group2 & 88.50 & 96.71 & 89.68 & 90.20 & 75.28 \\
group3 & 87.33 & 95.00 & 93.35 & 86.93 & 77.69 \\
group4 & 87.25 & 88.91 & 94.40 & 87.15 & 80.13 \\
arr(\%) & 88.39 & 92.31 & 91.20 & 88.15 & 78.87
\end{tabular}

It can be seen from the trial situation that all kinds of symbolic expressions can complete the most part of the text content prepared by researchers, among which the communication completion of emotional graphics symbols is the highest, reaching $92.31 \%$. This is due to a large number of emotional emoticons in popular social APP, which can be used by users skillfully. However, the communication completion of time and space graphics symbols is the lowest, only $78.87 \%$. Researchers think it is often used as a modified adverbial in the language and articles, so it is still difficult to carry out a complete dialogue only by the graphic symbols designed by this kind of vocabulary, and the setting of this category will be changed in the future.

\section{DISCUSSION}

In addition to voice, mood and other factors related to speech, other forms of perceivable nonverbal expression can be classified as "situation language". Situation language is characterized by the accessibility of thought expression, the visualization of emotional meaning, and the uniqueness of creating context. Network communication, as a mapping of real communication, can replace the lengthy text, and its function is equivalent to the situation language in real communication.Therefore, we found that in the construction and trial of this app, the graphic symbols in practical applications are completely hopeful to solve the intercultural communication barriers in a certain area of business activities. Although the design and application of graphic symbols are not mature at present, as long as a large number of experiments are carried out and the coding and decoding process from language to graphic symbols to language is emphasized in the experimental results, it is entirely possible to apply it to cross cultural business exchanges in the GMS.

\section{ACKNOWLEDGEMENT}

The design and construction of this app is completed by the technical support my tutor, Professor of the International Business School of Yunnan University of Finance and Economics and the theoretical support of Miss Menglan Qiu.

\section{REFERENCES}

[1] Hongxiu Fu. Research on Emoticons in the Text of Internet Instan Messaging: A Complementary Perspective[D]. South-central University For Nationalities, South-central University For Nationalities,2011.

[2] T. ROYCE. Visual-Verbal Intersemiotic Complementarity in the Economist Magazine(Doctoral Dissertation)[M]. The University of Reading, 1999.

[3] Xiao Li. The current situation and challenges of the development of e-commerce in GMS[J]. Inquiry Into Economic Issues, 2004(4):87-89.

[4] Yuhui Feng, Meiyun Hua. Research on Non-verbal Graphic Symbol Communication of Cross-Border e-Commerce. Digital Services and Information Intelligence. Springer Berlin Heidelberg, 2014: 251-263, Accession number: 20150400448188.

[5] Xue Zhang. The Use of Network Expression in Mobile Chat Software from Visual Culture[J]. Journal News Research,2015:105-120.

[6] Yuhui Feng, Menglan Qiu, Yan Li, Haiyang Yang. Cross-culture Business Communication by Emoji in GMS[J/OL].atlantis-press.com,2016.

[7] Lei Pang, Shoushan Li, Guodong Zhou . Chinese Micro-blog Sentiment Classification Based on Emotion Knowledge[J]. Computer Engineering,2012,38(13):156-158.

[8] ROYCE T. Multimodality in the TESOL classroom--exploring visual-verbal synergy[J]. 2002,36(2):191-205.

[9] Patricia Wallace. The Psychology of the Internet[M]. Beijing: China Light Industry Press, 2001:10-16. 